\title{
Erratum to: Elevated Levels of IL-18 in Plasma and Skeletal Muscle in Chronic Obstructive Pulmonary Disease
}

\author{
A. M. W. Petersen - M. Penkowa $\cdot$ M. Iversen • \\ L. Frydelund-Larsen $\cdot$ J. L. Andersen • \\ J. Mortensen $\cdot$ P. Lange $\cdot$ B. K. Pedersen
}

Published online: 13 November 2011

(C) Springer Science+Business Media, LLC 2011

\section{Erratum to: Lung (2007) 185:161-171 \\ DOI 10.1007/s00408-007-9000-7}

In the original publication of the article, there was an error regarding plasma interleukin (IL)-18. Though the values for plasma-IL-18 in the abstract are correct, the $p$-value in the abstract should read $p=0.05$. In the Results section regarding plasma cytokines, the $p$-value should be $p=0.05$. The data regarding the plasma concentration of IL-18 does not change our conclusions of the study. However, we have to emphasize that plasma IL-18 in COPD patients are only borderline significant $(p=0.05)$ from the healthy controls.

The online version of the original article can be found under doi: 10.1007/s00408-007-9000-7.

A. M. W. Petersen · M. Penkowa · L. Frydelund-Larsen · B. K. Pedersen

The Centre of Inflammation and Metabolism,

Department of Infectious Diseases, The Faculty of Health

Sciences, University of Copenhagen, Copenhagen, Denmark

A. M. W. Petersen · L. Frydelund-Larsen .

J. L. Andersen · B. K. Pedersen

Copenhagen Muscle Research Centre, Rigshospitalet,

The Faculty of Health Sciences, University of Copenhagen,

Copenhagen, Denmark

\section{Penkowa}

Department of Medical Anatomy, The Panum Institute,

The Faculty of Health Sciences, University of Copenhagen,

Copenhagen, Denmark

M. Iversen

Department of Cardiology, Rigshospitalet, The Faculty of Health

Sciences, University of Copenhagen, Copenhagen, Denmark
The correct version of Fig. 1a is given below.

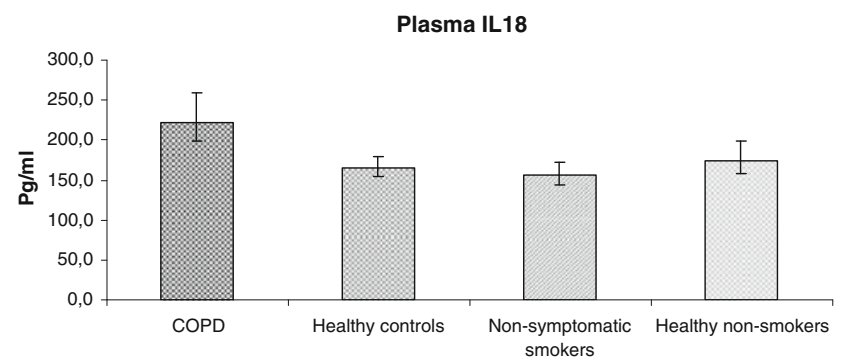

Fig. 1 (a) Plasma concentrations of interleukin (IL)-18 from COPD patients $(n=20)$, healthy controls $(n=20)$, nonsymptomatic smokers $(n=10)$, and healthy nonsmokers $(n=10)$. Values are given as geometric means and SEM

\footnotetext{
J. Mortensen

Department of Clinical Physiology and Nuclear Medicine,

Rigshospitalet, The Faculty of Health Sciences,

University of Copenhagen, Copenhagen, Denmark

P. Lange

Department of Pulmonary Diseases, Hvidovre Hospital,

The Faculty of Health Sciences, University of Copenhagen,

Copenhagen, Denmark

B. K. Pedersen $(\bowtie)$

Centre of Inflammation and Metabolism,

Rigshospitalet - Section 7641, Blegdamsvej 9, DK-2100

Copenhagen, Denmark

e-mail: bkp@rh.dk
} 